\title{
Affine Bernstein Problems Monge-Ampère Equations
}


This page intentionally left blank

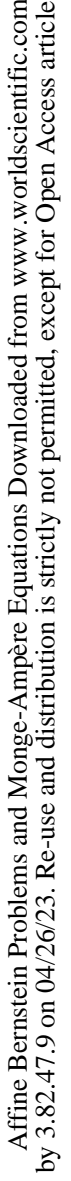




\section{Affine Bernstein Problems Monge-Ampère Equations}

An-Min Li (Sichuan University, China)

Ruiwei Xu (Henan Normal University, China)

Udo Simon (Technische Universität Berlin, Germany)

Fang Jia (Sichuan University, China) 


\section{Published by}

World Scientific Publishing Co. Pte. Ltd.

5 Toh Tuck Link, Singapore 596224

USA office: 27 Warren Street, Suite 401-402, Hackensack, NJ 07601

UK office: 57 Shelton Street, Covent Garden, London WC2H 9HE

\section{British Library Cataloguing-in-Publication Data}

A catalogue record for this book is available from the British Library.

\section{AFFINE BERNSTEIN PROBLEMS AND MONGE-AMPÈRE EQUATIONS}

Copyright (C) 2010 by World Scientific Publishing Co. Pte. Ltd.

All rights reserved. This book, or parts thereof, may not be reproduced in any form or by any means, electronic or mechanical, including photocopying, recording or any information storage and retrieval system now known or to be invented, without written permission from the Publisher.

For photocopying of material in this volume, please pay a copying fee through the Copyright Clearance Center, Inc., 222 Rosewood Drive, Danvers, MA 01923, USA. In this case permission to photocopy is not required from the publisher.

ISBN-13 978-981-281-416-6

ISBN-10 981-281-416-7

Printed in Singapore. 


\section{Preface}

Many geometric problems in analytic formulation lead to important classes of PDEs. Naturally, since all such equations arise in geometric context, geometric methods play a crucial role in their investigation. A classical example is given by the Euclidean Minkowski problem: the study of hyperovaloids with prescribed Gauß curvature in terms of the Euclidean unit normal field. For the history up to the early 70's see Pogorelov's monograph [77] from 1975 and the paper of Cheng and Yau [25] from 1976. The study of Minkowski's problem and the related regularity was essential for the understanding of certain Monge-Ampère type equations on the Euclidean sphere.

Our monograph is devoted to the interplay of global differential geometry and PDEs, more precisely to the study of some types of non-linear higher order PDEs; most of them have their origin in the affine hypersurface theories. Particular examples include the PDEs defining affine spheres and affine maximal hypersurfaces, resp., and the constant affine mean curvature equation.

Wide use of geometric methods in studying PDEs of affine differential geometry was initiated by E. Calabi and continued by A.V. Pogorelov, S.Y. Cheng-S.T. Yau, A.-M. Li, and, during the last decade, e.g. by N.S. Trudinger-X.J. Wang, A.-M. Li's school, and other authors.

The contributions of E. Calabi and S.Y. Cheng-S.T. Yau had a particularly deep influence on the development of this subject. According to the foreword in [25] this paper originated from discussions with E. Calabi and L. Nirenberg and results of both on the same topic; for further historical details and references we refer to [19], [20], [58], [76].

In problems involving PDEs of Monge-Ampère type it is often the case that the unknown solution is a convex function defining locally a nonparametric hypersurface for which it is possible to choose a suitable relative normalization and investigate the induced geometry. We refer to this process as geometric modelling. The choice of the normalization can be described in a unified and systematic manner in the context of relative hypersurface theory; for this theory see [58], [87], [88]. 
The next step involves derivation of estimates of various geometric invariants; a correct choice of a normalization is very important for successful completion of this step. Ultimately, such estimates are crucial for proving the existence and uniqueness, respectively, of solutions to the PDE.

In chapter 1 we start with a summary of basic tools; very good sources for that are the monographs [37], [50] and [58]. For a better understanding of the modelling techniques, in chapters 2 and 3 the authors give a selfcontained summary of relative hypersurface theory. Moreover, for the global study, we consider different notions of completeness in sections 4.2 and 5.9.

Chapters 4-6 are the central part of the monograph. They contain important PDEs from affine hypersurface theory: the PDEs for affine spheres, affine maximal surfaces, and constant affine mean curvature hypersurfaces. The PDE for improper affine spheres over $\mathbb{R}^{2}$ first was studied by Jörgens in the paper [49]; Calabi [19] extended the result to the dimensions $n \leq 5$, and finally Pogorelov to any dimension [76]. Later, Cheng and Yau extended Pogorelov's version and gave a simpler and more analytic proof in [25]; concerning this paper and Calabi's influence, see our remarks above. Nowadays, in the literature the Theorem is cited as Theorem of Jörgens-Calabi-Pogorelov. In section 4.4 we present the geometric Calabi-ChengYau proof for this theorem, [19], [25]. Afterwards we study a generalization of this theorem. As the proof of the generalization is relatively simple in dimensions $n \leq 4$, we use both proofs for a comparison of the geometric modelling procedure:

(i) In the proof of the Theorem of Jörgens-Calabi-Pogorelov we use Blaschke's normalization.

(ii) In the second example we give a proof of the generalization. Now we use a constant normalization of a graph and its induced geometry; to our knowledge it was first used by Calabi within this context.

Sections 4.5.5 and 4.6.2 present such comparisons of proofs with different modelling, emphasizing the interplay between the geometric model chosen and the PDE considered. In arbitrary dimension the proof of the extension of the Theorem of Jörgens-Calabi-Pogorelov is complicated, thus we carefully structure the proof as guideline for the reader (see section 4.5.7).

In chapter 5 we derive the Euler-Lagrange equation of affine maximal hypersurfaces. The topic of this chapter is given by different versions of the so called "Affine Bernstein Problem", in particular the "Affine Bernstein Conjectures" in dimension $n=2$. They are due to Chern and Calabi, resp., and were solved during the last decade. In 2000, Trudinger and Wang solved Chern's conjecture in dimension $n=2$ [91]; later, Li and Jia [52], and also Trudinger and Wang [92], solved Calabi's conjecture for two dimensions independently, using quite different methods. In section 5.7 we treat Calabi's Affine Bernstein Problem in dimensions $n=2$ and $n=3$. 
The final chapter studies constant affine mean curvature hypersurfaces. In dimension $n=2$ the problem was solved in case the constant is positive; in case the constant is zero we have again the "Affine Bernstein Problems". The case of negative constant mean curvature has been solved partially only, so far. For any bounded convex domain, we can construct a Euclidean complete affine hypersurface with negative constant affine mean curvature solving a boundary value problem for a fourth order PDE.

The monographs [5] and [37] give a good basis for the geometric theory of MongeAmpère equations. Our monograph gives a geometric method for the study of Monge-Ampère equations and fourth order nonlinear PDEs arising in affine differential geometry. There are recent related papers from A.-M. Li's school (e.g. [24]), and there are extensions to Kähler geometry and projective Blaschke manifolds [63]. Other interesting results concern global affine maximal surfaces with singularities, see e.g. [2], [3], [4], [34], [69].

The authors present three generations of geometers. U. Simon finished his doctoral thesis with K.P. Grotemeyer at the FU Berlin in 1965, and from his lectures he became interested in global differential geometry. U. Simon became a professor of mathematics at TU Berlin in 1970. A.-M. Li started his studies at Peking University in 1963, but because of the cultural revolution he could not finish his MS before 1982. Following a recommendation of S.S. Chern, he came as AvH fellow to the TU Berlin in 1986 the first time, and there he finished his doctoral examination with U. Simon, U. Pinkall and K. Nomizu. A.-M. Li has been a professor of mathematics at Sichuan University since 1986, successfully guiding research groups since then. A.-M. Li was also the advisor of F. Jia (PhD 1997) and R. Xu (PhD 2008) at Sichuan University, both are now professors themselves, F. Jia at Sichuan University (1997), R. Xu at Henan Normal University since 2008.

The homepages of our Chinese-German cooperation give some more details, for the momentary project see http://www.math.tu-berlin.de/geometrie/gpspde/.

Blaschke's interest in the global study of submanifolds was important for Chern's decision to go to Hamburg in 1934, and not to Göttingen. Their interest in global problems influenced the following generations. We aim to stimulate young geometers again.

Acknowledgements. The authors thank the following institutions for financial support that made possible joint work on the topic at Chengdu and Berlin, respectively: Alexander von Humboldt Stiftung (AvH), Deutsche Forschungsgemeinschaft (DFG), Dierks von Zweck Stiftung Germany, NSF China (10631050, 10926172, 10871136), RFDP, Sichuan University, TU Berlin and Henan Normal University. Moreover, TU Berlin made it possible that the authors could work together on this monograph in Berlin for several months in 2008 and 2009. 
We thank Mr. Min Xiong, Sichuan University, for a careful reading of our manuscript, and Vladimir Oliker from Emory University for very helpful discussions on the topic.

A.-M. Li, R. Xu, U. Simon, F. Jia

January 2010 


\section{Contents}

Preface $\quad$ v

1. Basic Tools 1

1.1 Differentiable Manifolds . . . . . . . . . . . . . . . . . 1

1.1.1 Manifolds, connections and exterior calculus . . . . . . . 1

1.1.2 Riemannian manifolds . . . . . . . . . . . . . . 4

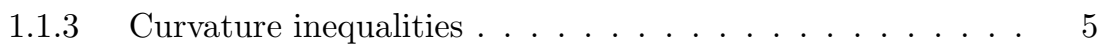

1.1.4 Geodesic balls and level sets . . . . . . . . . . . . 6

1.2 Completeness and Maximum Principles . . . . . . . . . . . . 7

1.2.1 Topology and curvature . . . . . . . . . . . . 7

1.2.2 Maximum principles..................... 7

1.3 Comparison Theorems . . . . . . . . . . . . . 8

1.4 The Legendre Transformation . . . . . . . . . . . . . . . . . 9

2. Local Equiaffine Hypersurfaces 11

2.1 Hypersurfaces in Unimodular Affine Space . . . . . . . . . . . . . . 11

2.1.1 The ambient space. . . . . . . . . . . . . . 11

2.1.2 Affine hypersurfaces . . . . . . . . . . . . 13

2.2 Structure Equations and Berwald-Blaschke Metric . . . . . . . . . 14

2.2.1 Structure equations - preliminary version . . . . . . . . . 14

2.2.2 Covariant Gauß equations - preliminary . . . . . . . . 16

2.3 The Affine Normalization . . . . . . . . . . . . . . . . . 16

2.3.1 The affine normal . . . . . . . . . . . . . 16

2.3.2 Affine shape operator and affine extrinsic curvature . . . . 18

2.3.3 The affine conormal . . . . . . . . . . . . . . 19

2.3.4 The conormal connection . . . . . . . . . . . . 21

2.3.5 Affine Gauß mappings ............... 21

2.4 The Fubini-Pick Form . . . . . . . . . . . . . . . . . 22

2.4.1 Properties of the Fubini-Pick form . . . . . . . . . . . 23

2.4.2 The Pick invariant . . . . . . . . . . . . . . . 23 
2.4.3 Structure equations - covariant notation . . . . . . . . 23

2.4.4 The affine support function . . . . . . . . . . . . . 24

2.5 Integrability Conditions . . . . . . . . . . . . . 24

2.5.1 Integration via moving frames . . . . . . . . . . . . 24

2.5.2 Covariant form of the integrability conditions . . . . . . 26

2.6 Fundamental Theorem . . . . . . . . . . . . . . . . . . 27

2.7 Graph Immersions with Unimodular Normalization . . . . . . . . . 27

2.8 Affine Spheres and Quadrics . . . . . . . . . . . . . . . 30

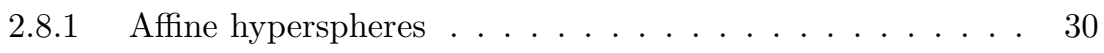

2.8.2 Characterization of quadrics ........... . . 31

3. Local Relative Hypersurfaces 33

3.1 Hypersurfaces with Arbitrary Normalization . . . . . . . . . . . 33

3.1.1 Structure equations . . . . . . . . . . . . . . 33

3.1.2 Fundamental theorem for non-degenerate hypersurfaces . . 35

3.2 Hypersurfaces with Relative Normalization . . . . . . . . . . . 35

3.2.1 Relative structure equations and basic invariants . . . . . . 36

3.2.2 Relative integrability conditions . . . . . . . . . . . 38

3.2.3 Classical version of the integrability conditions . . . . . . . 38

3.2.4 Classical version of the fundamental theorem . . . . . . . . 38

3.3 Examples of Relative Geometries . . . . . . . . . . . . . . . 39

3.3.1 The Euclidean normalization . . . . . . . . . . . . . 39

3.3.2 The equiaffine (Blaschke) normalization . . . . . . . . . 39

3.3.3 The centroaffine normalization . . . . . . . . . . . . 40

3.3.4 Graph immersions with Calabi metric . . . . . . . . . . 41

3.3.5 The family of conformal metrics $G^{(\alpha)}$. . . . . . . . . . . . 42

3.3.6 Comparison of different relative geometries . . . . . . . . 43

3.3.7 Different versions of fundamental theorems . . . . . . . . . 43

3.4 Gauge Invariance and Relative Geometry . . . . . . . . . . . . . 43

4. The Theorem of Jörgens-Calabi-Pogorelov 47

4.1 Affine Hyperspheres and their PDEs . . . . . . . . . . . . . . . . . 47

4.1 .1 Improper affine hyperspheres . . . . . . . . . . . . . . 47

4.1.2 Proper affine hyperspheres . . . . . . . . . . . . . 48

4.1.3 The Pick invariant on affine hyperspheres . . . . . . . . . . 49

4.2 Completeness in Affine Geometry . . . . . . . . . . . . . . . 50

4.2.1 Affine completeness and Euclidean completeness . . . . . . 50

4.2.2 The Cheng-Yau criterion for affine completeness . . . . . . 51

4.2.3 Proof of the Estimate Lemma . . . . . . . . . . . . 53

4.2.4 Topology and the equiaffine Gauß map . . . . . . . . . . 56

4.3 Affine Complete Elliptic Affine Hyperspheres . . . . . . . . . . . . 59

4.4 The Theorem of Jörgens-Calabi-Pogorelov . . . . . . . . . . . . . . 59 
4.5 An Extension of the Theorem of Jörgens-Calabi-Pogorelov . . . . . 61

4.5.1 Affine Kähler Ricci flat equation . . . . . . . . . . . . 61

4.5.2 Tools from relative geometry . . . . . . . . . . . 63

4.5.3 Calculation of $\Delta \Phi$ in terms of the Calabi metric . . . . . . 63

4.5.4 Extension of the Theorem of Jörgens-Calabi-Pogorelov proof for $n \leq 4 \ldots \ldots \ldots$. . . . . . . . . . . . . . . . . . 66

4.5.5 Comparison of two geometric proofs . . . . . . . . . 68

4.5.6 Technical tools for the proof in dimension $n \geq 5 \ldots 69$

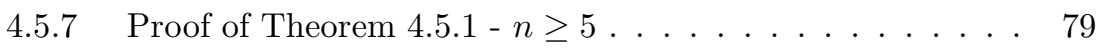

4.6 A Cubic Form Differential Inequality with its Applications . . . . . 82

4.6.1 Calculation of $\Delta J$ in terms of the Calabi metric . . . . . . 83

4.6.2 Proof of Theorem 4.6.2 . . . . . . . . . . . . . 85

5. Affine Maximal Hypersurfaces $\quad 89$

5.1 The First Variation of the Equiaffine Volume Functional . . . . . . 89

5.2 Affine Maximal Hypersurfaces . . . . . . . . . . . . . . . . . . . 92

5.2.1 Graph hypersurfaces ............... . . . . 92

5.2.2 The PDE for affine maximal hypersurfaces . . . . . . . . 95

5.3 An Affine Analogue of the Weierstrass Representation . . . . . . . 96

5.3.1 The representation formula . . . . . . . . . . . 96

5.3.2 Examples....................... 99

5.4 Calabi's Computation of $\Delta J$ in Holomorphic Terms . . . . . . . . 99

5.4 .1 Computation of $\Delta\left(J+\|B\|^{2}\right) \ldots \ldots \ldots$. . . . . . . . . . . . . 104

5.5 Calabi's Conjecture . . . . . . . . . . . . . 105

5.5.1 Proof of Calabi's Conjecture for dimension $n=2$. . . . 106

5.6 Chern's Conjecture . . . . . . . . . . . . . . 110

5.6.1 Technical estimates . . . . . . . . . . . . . . 112

5.6.2 Estimates for the determinant of the Hessian . . . . . . . . 114

5.6.3 Estimates for the third order derivatives . . . . . . . . . 121

5.6.4 Estimates for $\sum f_{i i} \ldots \ldots \ldots 126$

5.6.5 Proof of Theorem 5.6.2 . . . . . . . . . . . . 128

$5.7 \quad$ An Affine Bernstein Problem in Dimension 3 . . . . . . . . . . . . 131

5.7.1 Proof of Part I . . . . . . . . . . . . . . 131

5.7.2 Proof of Part II: Affine blow-up analysis . . . . . . . . . . 133

5.8 Another Method of Proof for some Fourth Order PDEs . . . . . . 138

5.9 Euclidean Completeness and Calabi Completeness . . . . . . . . . 144

6. Hypersurfaces with Constant Affine Mean Curvature 149

6.1 Classification . . . . . . . . . . . . . . 149

6.1.1 Estimates for the determinant of the Hessian . . . . . . . . 150

6.1.2 Proof of Theorem 6.1.1 . . . . . . . . . . . . 151

6.1.3 Proof of Theorem 6.1.2 . . . . . . . . . . . 160 
6.2 Hypersurfaces with Negative Constant Mean Curvature . . . . . . 161

6.2.1 Proof of the existence of a solution . . . . . . . . 165

6.2.2 Proof of the Euclidean completeness . . . . . . . . . . . . 169

Bibliography 